\title{
La pirogue et le grenier
}

Les déterminants techno-économiques de la transition aux paiements

The granary and the canoe. The techno-economic determinants of the transition to payments

\section{Christophe Darmangeat}

\section{(2) OpenEdition Journals}

Édition électronique

URL : http://journals.openedition.org/artefact/860

DOI : $10.4000 /$ artefact.860

ISSN : 2606-9245

\section{Éditeur :}

Association Artefact. Techniques histoire et sciences humaines, Presses universitaires du Midi

\section{Édition imprimée}

Pagination : 133-151

ISBN : 978-2-7535-7305-5

ISSN : 2273-0753

Référence électronique

Christophe Darmangeat, « La pirogue et le grenier », Artefact [En ligne], 6 | 2017, mis en ligne le 31 mai 2018, consulté le 05 mars 2020. URL : http://journals.openedition.org/artefact/860 ; DOI : https:// doi.org/10.4000/artefact.860 


\section{La pirogue et le grenier. Les déterminants techno-économiques de la transition aux paiements}

Christophe DARMANGEAT ${ }^{*}$

\section{Résumé}

Alain Testart a montré, il y a une trentaine d'années, que l'apparition des inégalités socio-économiques et de la richesse (définie par les paiements de mariage ou la compensation pour meurtre) n'est pas corrélée à l'agriculture, mais au stockage alimentaire. Cet article réexamine cette assertion à la lumière des rares sociétés à paiements qui, en raison de conditions écologiques particulières, ne pratiquent pas un tel stockage, en suggérant que la variable clé de la transition aux paiements est la production d'une catégorie particulière de biens.

Mots-clés : agriculture, environnement, inégalités, prix de la fiancée, stockage.

\section{Abstract. The granary and the canoe. The techno-eco- nomic determinants of the transition to payments}

About thirty years ago, Alain Testart showed that the birth of the first socio-economic inequalities and of wealth (defined altogether by marriage payments and compensation for murder) were not related to agriculture, but to food storage. This statement is investigated under the light of the few societies with payments which, due to specific ecological conditions, were not based on storage. We suggest that the key factor in the transition to payments is the production of a specific category of goods.

Keywords : agriculture, bridewealth, environment, inequalities, storage.

\footnotetext{
* Christophe Darmangeat est enseignant à l'université Paris-Diderot et chercheur en économie et anthropologie sociale (Sophiapol, Ladyss). Il s'est intéressé tout particulièrement à la théorie de l'évolution sociale, aux origines des inégalités de richesse ou de genre. Il a publié plusieurs livres et articles, dont Le communisme primitif n'est plus ce qu'il était (Smolny, 2012). Son blog: [http://cdarmangeat.blogspot. com.]. Contact : [cdarmangeat@gmail.com].
} 
Identifier les évolutions techniques qui auraient donné naissance aux inégalités socio-économiques est, depuis toujours, une des questions centrales de l'étude des sociétés. Selon la théorie la plus communément acceptée durant le $\mathrm{xx}^{\mathrm{e}}$ siècle, le pas décisif a été franchi avec l'invention de l'agriculture et de l'élevage lors de ce que Vere Gordon Childe appelait la "révolution néolithique ${ }^{1}$ ». Contrairement aux chasseurs-cueilleurs qui se caractérisent par un fort égalitarisme économique, les cultivateurs exhibent des inégalités de richesse plus ou moins marquées. Childe voyait là l'effet d'un "surplus » alimentaire que seule l'agriculture avait pu engendrer et qui était censé expliquer tant l'apparition de la division du travail que celle de l'exploitation. La théorie du surplus a suscité d'intenses discussions qui n'ont jamais abouti à un consensus ${ }^{2}$; mais c'est sur le plan empirique que le scénario proposé par V. G. Childe a reçu, il y a une trentaine d'années, un coup décisif.

Deux ensembles de sociétés mettent en effet à mal la corrélation supposée entre agriculture et inégalités économiques. Il s'agit, d'une part, des peuples de chasseurs-cueilleurs sédentaires qui, tout en ignorant l'agriculture et l'élevage (hormis celui du chien), étaient marqués par une forte stratification sociale. La côte nordouest de l'Amérique du Nord fournissait l'exemple classique de cette anomalie des chasseurs-cueilleur dits " complexes". Mais on rencontrait également l'anomalie inverse, avec ces nombreux cultivateurs chez qui régnait un assez strict égalitarisme - du moins entre les individus masculins -, tels les Indiens des basses plaines d'Amazonie ou l'ensemble anga des Highlands de Nouvelle-Guinée.
Alain Testart fut le premier à en déduire que la clé de l'existence des inégalités économiques ne se situait pas, en réalité, dans l'agriculture, mais dans le stockage alimentaire, les deux termes n'étant nullement synonymes ${ }^{3}$. Si des troupeaux représentent par nature une forme de stockage, l'agriculture n'implique le stockage que dans le cas de plantes saisonnières telles que les céréales; certains végétaux tropicaux, comme le manioc, peuvent en revanche être récoltés tout au long de l'année. Inversement, si le nomadisme des chasseurs-cueilleurs non éleveurs de chevaux ou de rennes prohibait l'existence de stocks significatifs, la sédentarité qui prévalait chez les chasseurs-cueilleurs de la côte nord-ouest était étroitement associée au stockage à grande échelle $\mathrm{du}$ saumon, leur principale ressource (fig. 1). Capturé en grandes quantités à la période de l'année où il remontait les cours d'eau en bancs serrés, celui-ci était fumé afin d'être consommé durant la morte-saison.

A. Testart insistait d'ailleurs moins sur l'apparition des inégalités économiques stricto sensu - sans doute celle-ci est-elle bien difficile à cerner - que sur la naissance des paiements, c'est-à-dire de la richesse ${ }^{4}$. En effet, les sociétés sans stockage sont celles où les compensations sociales qui découlent pour l'essentiel du mariage et des dommages infligés à des tiers donnent exclusivement lieu à des obligations d'ordre personnel : un certain temps de travail pour les futurs beauxparents dans le cas du « service pour la fiancée ", des blessures physiques dans les diverses variantes de la loi du talion. Avec le stockage alimentaire, ces mêmes obligations prennent dorénavant la 


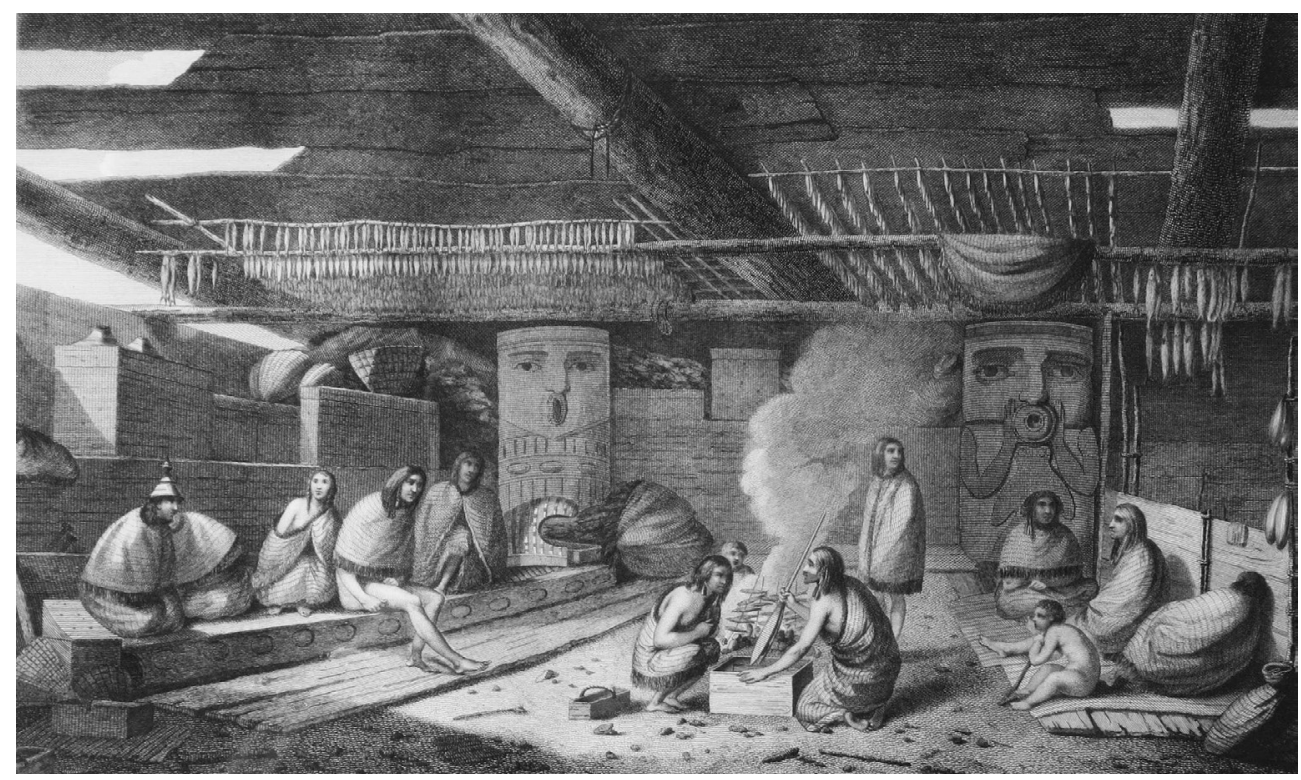

Figure 1.- « Intérieur d'une maison nootka », gravure de John Webber (1784).

On remarque les stocks de poissons séchés, suspendus au plafond.

forme de paiements en biens matériels : " prix de la fiancée » lors du mariage, " prix du sang » (ou wergeld) pour compenser des dommages. Pour A. Testart, l'avènement de la richesse constituait le plus grand bouleversement jamais connu par les rapports sociaux, au même titre et sans doute même davantage que l'apparition ultérieure des classes.

Cette perspective nouvelle a représenté un progrès considérable dans la théorie de l'évolution sociale. En reformulant les conditions du passage aux paiements et par là même à l'affirmation des inégalités de richesse, elle incluait dans les régularités d'une loi la presque totalité des configurations jusque-là considérées, faute de mieux, comme anormales. Un cas de figure résiste toutefois à cette réintégration : celui de sociétés qui, bien que non stockeuses, étaient tout à la fois sédentaires et structurées par la richesse. Une telle possibilité ne peut se rencon- trer que dans des milieux particulièrement propices : les ressources doivent y être disponibles sans discontinuer tout au long de l'année, soit qu'elles ne connaissent pas de creux saisonnier, soit que différentes ressources saisonnières se succèdent de manière à permettre un approvisionnement continu. Or cette éventualité est incarnée par quelques cas ethno-archéologiques susceptibles d'être étudiés et qui soulèvent par conséquent une interrogation d'ordre théorique : ces sociétés qui violent la corrélation générale entre stockage alimentaire et transition aux paiements, posent à la thèse d'Alain Testart un défi auquel, à notre connaissance et bien qu'il en ait implicitement posé les termes, il n'a jamais tenté de répondre.

C'est à ce problème qu'est consacré cet article $^{5}$, qui commencera par examiner les deux principaux cas connus de société à richesse caractérisés par une 
" sédentarité en raison de conditions écologiques favorables ${ }^{6} »$. Une fois leur validité confirmée, on tentera de reformuler de manière plus adéquate les conditions techno-économiques du passage aux paiements.

\section{Les cas ethno-archéologiques}

\section{Les Calusa}

Le premier exemple est celui des Calusa, des pêcheurs jadis installés sur la côte occidentale de la Floride. L'ethnographie de cette société se limite à quelques témoignages émanant de leurs premiers contacts avec les Espagnols, au début du xvie siècle. Plusieurs naufragés, en particulier, avaient été recueillis sur leurs terres et retenus en captivité (dont Hernando de Escalante Fontaneda, qui y demeura durant dix-sept ans) et jouèrent ensuite un rôle durant les relations brèves mais intenses que ce peuple entretint avec les conquistadores dans les années 1560. Les Espagnols se retirèrent alors de la région et les Calusa disparurent au début du xvIII ${ }^{\mathrm{e}}$ siècle, victimes tant des armes à feu de leurs voisins Creek que des infections apportées par les Européens. Dans une période plus récente, l'archéologie est venue ajouter quelques informations précieuses, mais qui laissent néanmoins dans l'ombre certains points essentiels (fig. 2).

\section{La subsistance}

Tous les indices concordent sur le fait que cette population de quelques milliers de membres, qui connaissait la poterie depuis au moins deux millénaires, ne cultivait presque aucune plante à l'exception possible de la courge. Le maïs en particulier, céréale de base sur l'ensemble $\mathrm{du}$ continent américain, était absent, ce que confirment les plus récentes analyses squelettiques et archéobotaniques ${ }^{7}$. Les Calusa se nourrissaient de poisson, très abondant sur cette côte, de mollusques, de tortues et de mammifères marins, ainsi que de plantes terrestres sauvages.

$\mathrm{Ni}$ dans les récits des témoins, ni dans les données étudiées par les archéologues, on ne trouve trace d'un quelconque stockage alimentaire. Au demeurant, on ne voit guère quel en aurait été l'intérêt : le milieu littoral et subtropical fournit tout au long de l'année d'abondantes ressources.

\section{Une stratification sociale poussée}

La société calusa était fortement stratifiée. À sa tête se trouvait un chef, résident du village principal, auquel des dizaines d'autres villages devaient tribut sous forme de nourriture, de plumes et de peaux ${ }^{8}$. Fait assez banal en Amérique, le chef avait le privilège de la polygamie et les villages lui faisaient allégeance notamment en lui procurant une épouse. Sa personne était magnifiée par des signes extérieurs de supériorité : son front était orné d'or et de perles et il siégeait sur un trône. Pour le saluer, les subordonnés plaçaient leurs paumes vers le haut et le chef les recouvrait des siennes ${ }^{9}$, un geste qui n'est pas sans évoquer celui par 


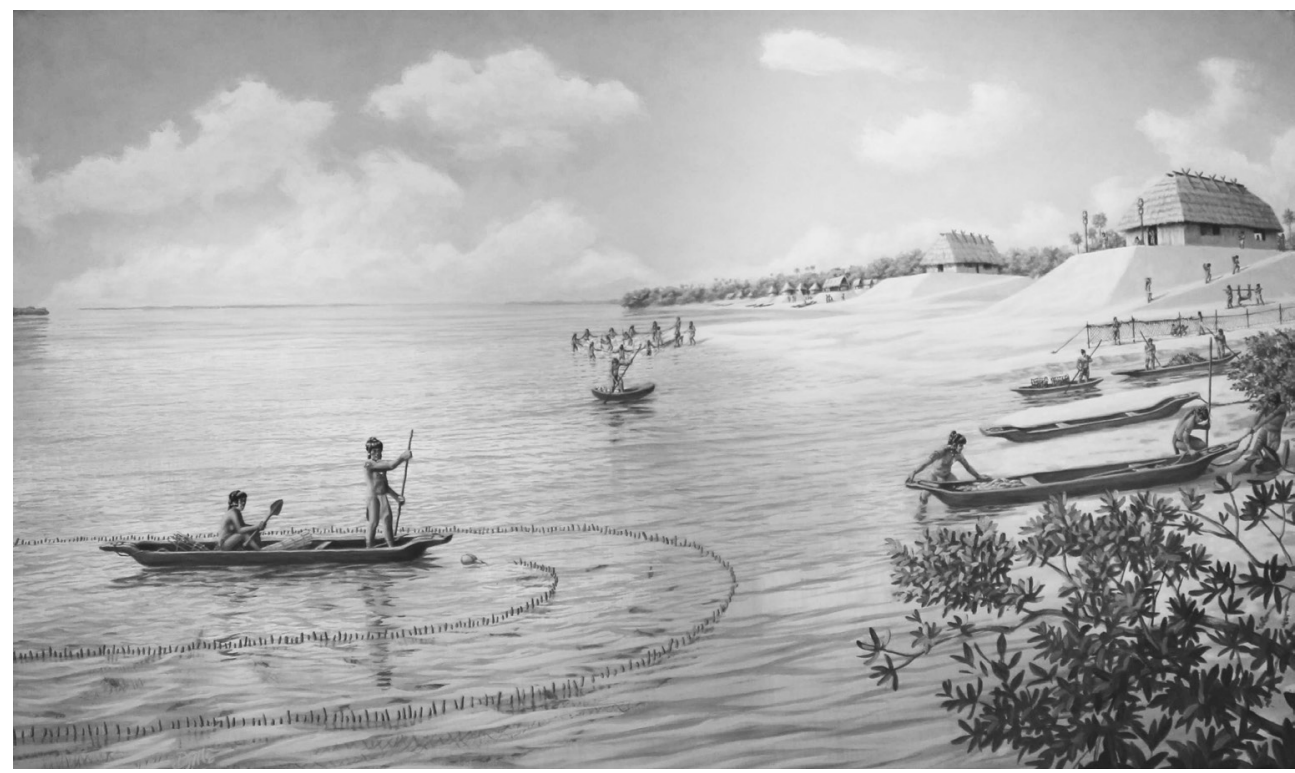

Figure 2. - « Reconstitution d'un village Calusa », dessin de David Meo.

lequel le vassal du Moyen Âge prêtait hommage à son suzerain. Lorsqu'il décédait, certains des subalternes étaient mis à mort à seule fin de l'accompagner dans la tombe, une pratique caractéristique de sociétés non étatiques nettement hiérarchisées $^{10}$. Enfin, entre ce chef et la masse des gens ordinaires, une strate intermédiaire de "nobles » occupait des fonctions politico-religieuses sur lesquelles nous sommes assez mal renseignés. Au bas de l'échelle sociale, se trouvait un groupe de dépendants, captifs de guerre, possiblement esclaves.

Même si les lacunes de nos informations laissent subsister d'importantes interrogations - en particulier dans quelle mesure leurs structures sociales pouvaient être la conséquence de l'arrivée des premières marchandises espagnoles -, les Calusa constituent un cas probant de société sans stockage et à richesse.

\section{Les Asmat}

Cet ensemble d'environ 30000 membres $^{11}$, dont les institutions sociales sont assez bien documentées, constitue le second exemple de société à " sédentarité en raison de conditions écologiques favorables ». Installé sur une zone marécageuse de la côte méridionale de la Nouvelle-Guinée, il ne fut guère en contact avec les Occidentaux avant les années 1950; il était alors encore peu influencé par leur commerce.

\section{La subsistance}

La nourriture des Asmat provenait de la pêche, mais surtout du palmier sagoutier (Metroxylon sagu), susceptible de fournir de la farine tout au long de l'année. La totalité des premiers observateurs assurent que les Asmat se bornaient à récolter le sagoutier sauvage sans pratiquer aucune forme de culture, ni pour cette plante, ni pour d'autres. 
C'est seulement à la fin des années 1960 que quelques arbres commencèrent à être plantés dans l'enceinte du village ${ }^{12}$ - ne livrant cependant qu'une part négligeable de la farine récoltée -, ou qu'apparurent quelques " jardins rudimentaires ${ }^{13}$ » où la plupart des espèces cultivées avait été introduite par les Européens.

Paul Roscoe, dans une belle synthèse, souligne que, dans l'ensemble de la Nouvelle-Guinée, « ce qui est particulièrement révélateur dans [les] différences de complexité culturelle est l'absence apparente de toute corrélation significative avec le degré de dépendance vis-à-vis de l'agriculture ${ }^{14}$ ». Les Asmat, avec leur alimentation exclusivement ou presque exclusivement sauvage, connaissaient ainsi des inégalités économiques nettement plus marquées que certaines tribus de la même zone qui pratiquaient l'agriculture.

Tout comme pour les Calusa, et avec les mêmes difficultés de méthode, se pose la question de l'absence de stockage. Celui-ci n'apparaît guère nécessaire, la production du sagoutier néo-guinéen n'étant pas saisonnière. On lit néanmoins que la farine, une fois préparée, peut se conserver plusieurs semaines, voire plusieurs mois ${ }^{15}$. D'autres éléments suggèrent que, pour des raisons purement sociales, la récolte de sagou était sujette à de brusques à-coups. Les longs cycles cérémoniels donnaient lieu à diverses distributions de nourriture dans la maison des hommes et, lors de leur conclusion, à un grand festin ${ }^{16}$. Afin de pourvoir à ces libéralités, ceux qui les dirigeaient conviaient les autres membres de la maison des hommes sur leurs parcelles afin d'y couper des arbres.
Trenkenshuh estime qu'en 1969, la fête du Udjung Sagu, rassemblant plus d'un millier de participants, avait donné lieu à l'abattage simultané de mille cinq cents palmiers $^{17}$ ! On ne saurait cependant déduire de ces seuls faits l'existence d'un stockage significatif dont aucun observateur n'a jamais fait état, pas plus que des dispositifs matériels qui n'auraient pas manqué de l'accompagner. Plus encore que dans le cas des Calusa - nos informations sur les Asmat étant infiniment plus précises -, on peut donc tenir pour certain que leur économie ne reposait pas sur le stockage.

\section{Une société à richesse}

La société asmat était bien moins stratifiée que ne pouvait l'être la société calusa. Elle n'en restait pas moins marquée par le rôle des paiements et de la richesse qui avaient suscité l'émergence d'inégalités palpables.

Le mariage pouvait s'effectuer par simple échange de conjoints; c'était le cas lorsqu'il intervenait, à la suite d'un accord entre les parents, entre individus du même yew (du nom des subdivisions villageoises, qui ne recouvraient qu'assez approximativement des relations de parenté; dans le village le mieux connu au cours des années 1960, on en comptait cinq pour quelques centaines $\mathrm{d}^{\prime}$ habitants $\left.{ }^{18}\right)$. Ce type de situation correspondait peut-être à la moitié des mariages. Dans tous les autres cas, soit que les époux appartiennent à des yew différents, soit que l'union ait été nouée contre le consentement des parents, sa validation supposait le versement d'un prix de la fiancée : haches de pierre, colliers de dents de chien, coquillages, lances et flèches, dagues d'os, oiseaux 
de paradis, plumes de casoar, capes de peaux de couscous, etc. ${ }^{19} \mathrm{Ce}$ paiement était si élevé qu'il était généralement échelonné ${ }^{20}$; dans le cas où le fiancé était trop pauvre pour s'en acquitter, il devait effectuer, à titre de substitution, un temps de service pour le compte de ses beaux-parents ${ }^{21}$.

Les Asmat connaissaient également le " prix du sang ", ou wergeld, qui intervenait lorsque l'affaire mettait aux prises les membres d'un même village ou de villages apparentés ${ }^{22}$. On note avec intérêt que " l'importance relative dans le village des deux individus impliqués au départ dans la dispute, ainsi que de leurs parents, entre également en considération. Un homme important peut exiger une forte indemnité et faire en sorte de l'obtenir ${ }^{23}$ ».

La place prise par la richesse se manifestait dans les conditions d'accession au leadership. Dans cette société marquée par d'incessantes expéditions de chasse aux têtes, le succès dans ces entreprises représentait la première condition de la réussite sociale :

« Dans la société asmat, tout prestige, et par conséquent toute autorité, découle en dernière instance des accomplissements martiaux. Il est impossible d'être un homme d'un statut socialement considéré sans avoir ramené quelques têtes. Une grappe de crânes accrochée au poteau de l'entrée est une mesure du statut ${ }^{24}$.»

Plusieurs observateurs ont toutefois souligné que l'accession au leadership supposait également de posséder une assise économique suffisante pour effec- tuer de vastes distributions de nourriture ${ }^{25}$. Cette nécessité se manifestait avec éclat à l'occasion de la fabrication des bisj pole, ces mâts gravés emblématiques des Asmat (fig. 3). Le commanditaire de l'entreprise devait en effet avoir les moyens de nourrir les graveurs durant tout le temps de leur office, soit deux ou trois mois. Ce n'était là que la moindre partie des frais : l'inauguration de l'ouvrage donnait lieu à une fête où étaient régalés non seulement les membres du village, mais des invités de villages voisins, si bien qu'elle pouvait rassembler deux mille convives ${ }^{26}$. Même si, dans ces dépenses, le leader accompli ou putatif était assisté de ses proches parents ou d'hommes qu'il avait attirés dans son sillage, il devait pour y faire face être luimême en état de mobiliser d'importantes ressources en sagou et en main-d'œuvre. Une telle mobilisation impliquait la polygynie : seules les femmes récoltaient le sagou et, à leur mariage, les droits qu'elles détenaient sur des zones de palmiers étaient transférés à leurs maris (un facteur qui contribuait à la lourdeur du prix de la fiancée ${ }^{27}$ ). Les leaders asmats étaient donc de grands guerriers polygynes, dont certains comptaient près $d^{\prime}$ 'une vingtaine d'épouses ${ }^{28}$.

Ainsi, pour être complète, l'ascension sociale supposait une double conversion : de la réussite martiale en réussite matrimoniale, et de celle-ci en richesse susceptible d'intervenir dans des paiements ou des distributions ostentatoires :

«Il faut souligner que l'équation entre, d'un côté, l'exploit guerrier et, de l'autre, l'abondance d'épouses et de surplus de sagou, est extrêmement importante. C'est par cette 


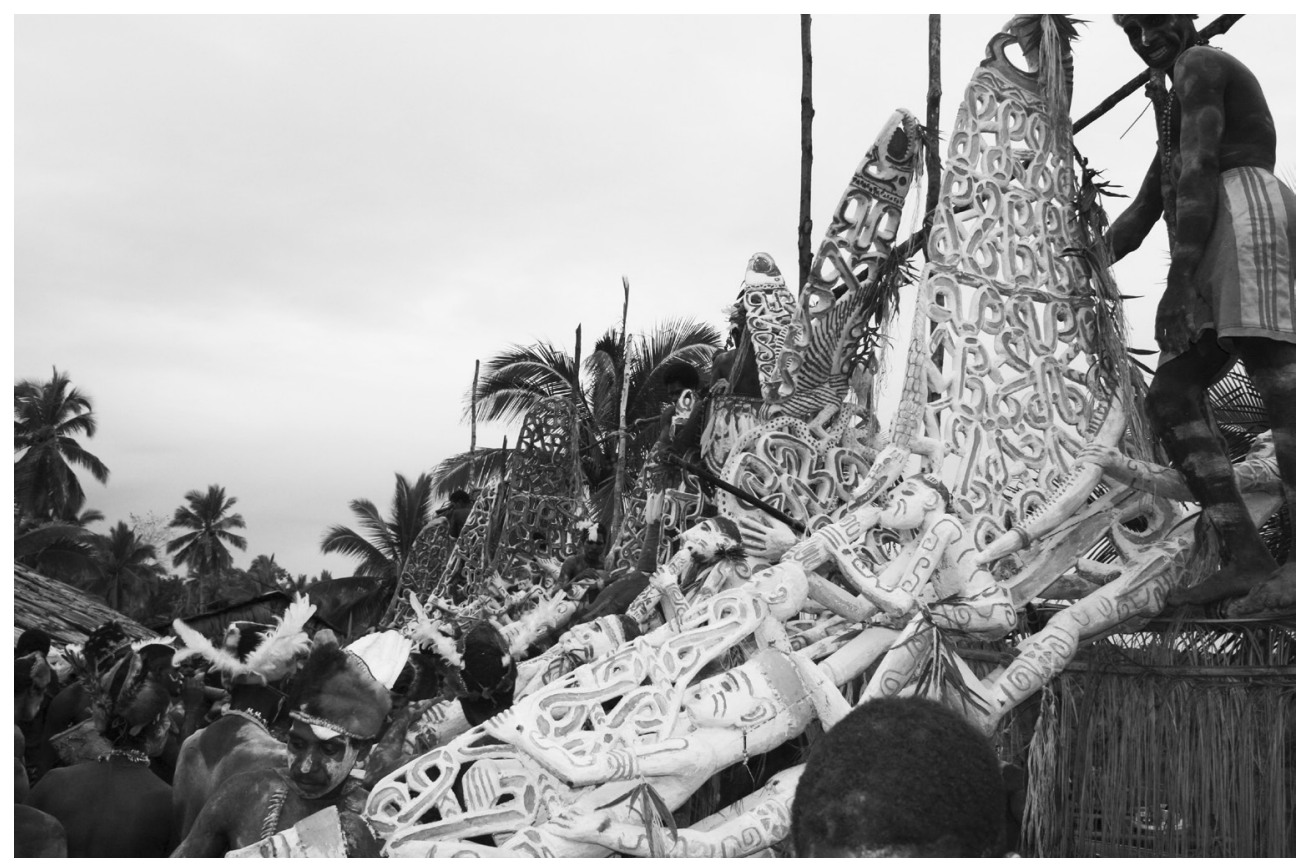

Figure 3. - « Une cérémonie d'inauguration de bisj pole (mâts gravés) », photographie de 2007, Wikipedia.

équation que l'exploit guerrier se voit transformé en capacité à procéder à des distributions massives de nourriture, et par conséquent en pouvoir politique ${ }^{29}$.»

Peut-être est-il difficile de suivre A. Testart lorsqu'il range la société asmat dans la vaste catégorie des "ploutocraties ostentatoires ${ }^{30}$ ", aux côtés par exemple des célèbres sociétés à Big Men manipulateurs de richesses. Bien que cette discussion dépasse le cadre de cet article, sans doute faudrait-il considérer comme un type à part entière ces formes sociales intermédiaires où le rôle bien établi de la richesse se combine à des institutions et des pratiques caractéristiques du monde achrématique. Pour rester dans l'aire néo-guinéenne, les Asmat se rapprochent sans doute davantage de ces sociétés « à leaders » des Highlands, elles aussi analysées par Pierre Lemonnier ${ }^{31}$ comme des formes intermédiaires. Quoi qu'il en soit, l'existence et la magnitude des paiements, conjointement au rôle non hégémonique, mais indéniable, de la richesse dans la structuration sociale, valide le cas asmat comme un second contre-exemple au rôle exclusif du stockage dans cette évolution. 


\section{Quel fondement techno-économique des paiements?}

Le problème évident que ces « sociétés sédentaires en raison de conditions écologiques favorables " posent à la thèse selon laquelle la richesse procèderait $\mathrm{du}$ stockage alimentaire ne peut être résolu que de deux manières. La première consisterait à considérer la valeur explicative du stockage comme nulle et non avenue, voire, dans une version encore plus radicale, à proclamer la faillite du programme matérialiste et à rejeter toute possibilité de fonder l'évolution des structures sociales sur la sphère technoéconomique. Une telle attitude reviendrait, selon l'expression consacrée, à jeter l'enfant avec l'eau du bain. Si elle admet certes quelques exceptions, l'adéquation entre richesse et stockage alimentaire se vérifie dans un très grand nombre de cas et sa découverte a représenté une avancée scientifique majeure. Il ne s'agit donc pas de récuser en bloc la thèse du stockage, mais de l'élargir afin d'y intégrer ce qui apparaît de prime abord comme des exceptions.

Pour avancer d'un pas dans cette direction, remarquons tout d'abord que celles-ci n'existent que dans un seul sens : on connaît quelques sociétés où la richesse existe sans stockage, mais il n'existe, semble-t-il, pas de société où le stockage existe sans la richesse. Les sociétés pratiquant le stockage alimentaire représentent donc un sousensemble (très majoritaire) des sociétés à richesse. Dès lors, l'hypothèse la plus simple consiste à considérer le stockage alimentaire comme un cas particulier (quoique très fréquent) de l'élément techno-économique qui détermine le passage à la richesse.

\section{Une hypothèse alternative : les biens $W$}

Cet élément consiste en la présence significative de biens meubles, durables, et dont la fabrication ou l'acquisition exige une quantité importante de travail effectué de manière individuelle (ou en groupes restreints). Dans la suite de ce texte, pour plus de commodité, ces biens seront appelés « biens $W$ », cette lettre se trouvant être, en anglais, à la fois l'initiale du travail (work) et de la richesse (wealth).

Dans un travail précédent ${ }^{32}$, nous avions déjà été amené à envisager un lien entre le travail investi dans les stocks alimentaires et la transition aux paiements. Il était en effet frappant qu'A. Testart, tout en ayant consacré de nombreuses pages aux prestations matrimoniales, puisse avouer ne pas être en mesure d'expliquer la nature exacte de la causalité entre ces deux éléments ${ }^{33}$. Or, sur un plan purement logique, l'apparition, à un certain niveau d'évolution technique, de productions exigeant un temps de travail important constitue une explication aussi simple que convaincante de la transition vers les paiements de mariage (et, par un lien logique depuis longtemps repérés ${ }^{34}$, avec les compensations pour meurtre). Qu'est-ce en effet que le passage au prix de la fiancée, sinon la reconnaissance par la société de l'équivalence entre un temps de travail prolongé (ce que sont le service temporaire 
ou les prestations viagères) et certains biens matériels? Et comment cette reconnaissance sociale ne procèderait-elle pas nécessairement de l'existence, dans la réalité, de biens représentant une grande quantité de travail?

\section{Les biens W chez les Calusa et les Asmat}

Nos informations sur les Calusa sont trop fragmentaires pour identifier avec une probabilité raisonnable quels produits pourraient y avoir représenté des biens $W$. Les Asmat, en revanche, connaissent au moins deux productions possibles de ce type : celle des haches de pierre et celle des canoës.

Il est assez difficile de déterminer la mesure dans laquelle les premières satisfaisaient à la définition des biens $W$. Leur fabrication ne paraît pas avoir exigé un temps de travail très important; comme tout peuple vivant dans une forêt peu transformée, les Asmat n'en polissaient que le tranchant, une opération qui ne nécessitait que quelques heures ${ }^{35}$. Toutefois, les habitants des zones côtières étaient dépourvus de matière première; les haches représentaient donc un précieux, et sans doute coûteux, bien d'importation ${ }^{36}$.

Quant aux canoës, ils possèdent toutes les caractéristiques requises pour entraîner le passage aux paiements. Leur taille était très variable : ceux destinés aux enfants mesuraient deux mètres; les canoës d'usage courant, pour la pêche et la récolte du sagou dans les marais, entre sept et quatorze mètres. Quant aux vaisseaux de guerre, ils pouvaient atteindre vingt mètres. L'embarcation était fabriquée d'une seule pièce à partir d'un tronc adéquat. Tout commençait par le repé- rage, puis l'abattage de l'arbre, choisi dans une espèce de dureté moyenne. On procédait ensuite à un premier évidage sur place; l'ébauche était ensuite amenée au village - le moment où elle devait être hissée hors de l'eau était le seul où une force de travail collective était impliquée -, et c'est près du lieu d'habitation que la finition se poursuivait jusqu'à son terme.

Eyde n'évalue pas précisément le temps passé à leur fabrication; pour l'essentiel, on ne se consacrait pas à celle-ci à temps plein, mais en plus des activités quotidiennes de pêche ou de récolte du sagou. Il faut donc se contenter de l'indication selon laquelle la réalisation d'un grand canoë pouvait exiger plus de deux mois de travail ${ }^{37}$. Dans cette zone marécageuse, et malgré le soin apporté à leur conservation, la durée de vie des embarcations n'excédait pas deux à trois ans et « la fabrication des canoës est probablement le travail le plus lourd effectué par les hommes. Dans un village asmat, on peut entendre le bruit sourd des haches presque chaque après-midi ${ }^{38}$. »

Or ces observations datent d'une époque où les haches de métal avaient entièrement remplacé les traditionnelles haches de pierre. D'après diverses estimations, l'économie de temps de travail apporté par les premières sur les secondes était de l'ordre des trois quarts, et davantage pour les très gros arbres - précisément ceux qui représentaient la matière première des canoës asmat ${ }^{39}$. Tout indique donc que la charge que représentait cette activité, déjà lourde dans les années 1960, devait être jadis considérablement plus élevée. Au bout du compte, tant par les efforts nécessaires à sa fabrication que par son rôle 
irremplaçable dans un environnement marécageux, le canoë représentait « la propriété manufacturée la plus importante des Asmat ${ }^{40}$ ».

Objectera-t-on que le canoë ne saurait expliquer le passage au prix de la fiancée, du fait qu'il ne faisait pas partie des biens qui le composaient? Cet argument peut être écarté pour deux raisons. En premier lieu, sur le plan théorique, rien n'oblige la composition du prix de la fiancée observée dans le présent ethnologique à être identique à celle qui prévalait lors de son institution. Ensuite, sur un plan empirique, et même si les informations déjà citées de $\mathrm{F}$. Trenkenshuh ne le mentionnent pas, il semble que chez les Asmat, le canoë faisait partie des compensations matrimoniales, en dehors même des situations qui donnaient lieu à un prix de la fiancée. C'est en tout cas ce que suggère Eyde, lorsqu'il écrit que la fabrication du canoë constitue " [...] l'un des principaux devoirs d'un homme envers les frères et le père de sa femme [...] un homme doit fournir un canoë aux frères ou au père de sa fiancée avant le mariage $\mathrm{e}^{41} »$.

\section{Biens W produits, biens W importés}

L'hypothèse des biens $W$ ne rend pas seulement compte du passage aux paiements dans des économies où ces biens voient le jour à la suite d'évolutions internes. Elle explique également les situations où cette transition s'est effectuée consécutivement à leur arrivée depuis l'extérieur. Cette idée est illustrée par le cas des Jivaro, fort bien décrit par Michael Harner dans les années 1950.

Les Jivaro font partie de ces nombreux chasseurs-cultivateurs non stockeurs
d'Amazonie chez lesquels, traditionnellement, les paiements n'existaient pas. Le mariage impliquait un temps de service pour le gendre, et la seule manière de compenser un meurtre était de tuer en retour. Cette absence de paiements peut être corrélée à la relative modestie matérielle des sociétés amazoniennes et à leur caractéristique bien connue d'être "principalement orientées vers la production des personnes, et non des biens matériels; c'est-à-dire qu'elles ne se concentrent pas sur la production des objets par le travail, mais sur celle des personnes par un travail rituel et symbolique $^{42}$ ». Or, l'arrivée de biens industriels par peuples voisins interposés, en particulier celle des machettes à lame de métal et des fusils, avait commencé à modifier cet état de fait (fig. 4). Ainsi, un prétendant pouvait dorénavant se voir dispensé du temps de service chez son beau-père s'il lui fournissait un fusil ${ }^{43}$. La cession d'un fusil pouvait également tenir lieu de compensation pour meurtre

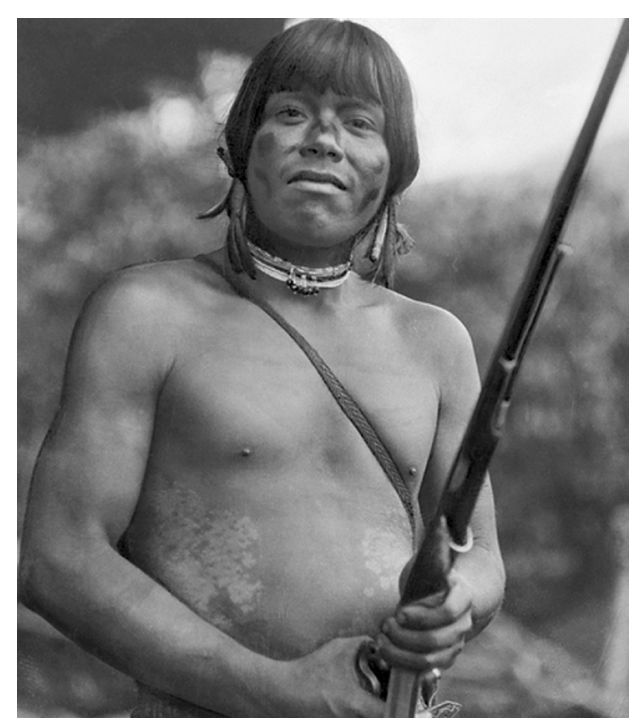

Figure 4. - Un Jivaro photographié avec sa carabine, en 1921, par H. E. Anthony. 
et mettre fin à une vendetta ${ }^{44}$. Ces informations traduisent clairement l'amorce d'une transition vers le prix de la fiancée et le wergeld, suite à l'arrivée d'au moins un instrument - le fusil - répondant à la définition d'un bien $W$; la différence avec le cas asmat est que le travail, chez les Jivaro, n'était pas directement investi dans sa production : il devait produire la contrepartie nécessaire à l'acquisition par l'échange de cet article d'importation.

\section{Deux questions supplémentaires}

\section{Les stocks alimentaires sont-ils des biens $\mathrm{W}$ ?}

Jusqu'ici, on a tenu pour acquis que les stocks alimentaires formaient un sous-ensemble des biens $W$, tout comme les sociétés pratiquant le stockage forment un sous-ensemble des sociétés à paiements et inégalités. Or, les deux propositions ne sont pas logiquement équivalentes. On pourrait fort bien imaginer que les stocks alimentaires doivent être exclus du périmètre des biens $W$-et, par conséquent, qu'ils soient incapables de provoquer par eux-mêmes la transition aux paiements - tout en étant, toujours ou le plus souvent, associés à certaines conditions technico-économiques qui, pour leur part, détermineraient cette transition. Autrement dit, on ne peut écarter a priori la possibilité que les stocks alimentaires soient corrélés aux paiements, mais qu'ils n'en constituent pas une cause.

Pour évaluer cette hypothèse, les faits ne nous aident guère; à notre connaissance, il n'existe aucune société où l'on ait observé à la fois un stockage significatif et une absence de paiements; faute de cet argument fort en défaveur de la causalité des biens alimentaires dans les paiements, on ne peut recourir qu'au seul raisonnement afin de tenter de faire la lumière sur ce point.

Même s'il ne posait pas exactement le problème en ces termes, A. Testart l'abordait dans un article posthume et inachevé ${ }^{45}$. Après avoir envisagé que le poisson séché ait représenté le point de passage vers le prix de la fiancée, il semblait écarter cette possibilité avec différents arguments. En premier lieu, il avançait l'impossibilité de fournir en une livraison unique l'équivalent d'une vie de travail. Mais cette objection n'a de sens que dans le cas où le prix de la fiancée remplace des obligations viagères et non un simple temps de service; ensuite, et surtout, elle pourrait être déclinée à l'identique pour n'importe quel autre bien. Or, le prix de la fiancée est une réalité, et un argument qui conduit à conclure à l'impossibilité de son instauration est nécessairement fautif. Plus intéressante est l'observation selon laquelle la nourriture, hormis sous forme de bétail, ne représente jamais la part principale du prix de la fiancée. A. Testart attribuait ce fait à ses caractéristiques matérielles qui en font une monnaie de piètre qualité : outre que la durabilité d'un stock alimentaire est rarement très élevée, la nourriture cristallise généralement dans un volume donné une quantité de travail relativement faible. Le poisson séché, tout comme la presque totalité des produits alimentaires, représente donc une "petite coupure » : c'est une menue monnaie, peu propice à régler une forte somme telle que, dans la plupart des cas, le prix de la fiancée. 
Cependant, nos propres piécettes ont beau être de la menue monnaie, elles sont de la monnaie tout de même et l'on peut légalement les utiliser pour régler ses achats - quitte à faire froncer les sourcils du vendeur. Ainsi, l'argument d'A. Testart explique pourquoi le poisson séché, de par ses caractéristiques physiques, est rarement utilisé dans les paiements dans la mesure où existent corrélativement d'autres biens, plus qualifiés pour ce rôle. Il ne démontre pas que le poisson (ou tout autre aliment stocké), s'il avait été le seul bien $W$ disponible, aurait été incapable d'entraîner le passage aux paiements; au demeurant, A. Testart lui-même termine son raisonnement par une formulation plus prudente qu'il ne l'inaugure. S'il commence par affirmer que " tout s'oppose s6 $^{46}$ au scénario dans lequel les stocks alimentaires provoquent le passage aux paiements, il conclut en écrivant que " tous les peuples, dans le cas où ils auraient vécu une phase durant laquelle il y eut un prix de la fiancée en nourriture, ont remplacé cette nourriture par des biens - manufacturés ou pas - mais en tout cas rares et difficiles à obtenir : de ce fait, ces biens étaient plus faciles à manipuler ${ }^{47}$ ». $C^{\prime}$ est là, on en conviendra, une idée tout à fait différente.

Plutôt que mettre en doute le fait que les stocks alimentaires représentent effectivement des biens $W$ et donc qu'ils possèdent la capacité d'entraîner le passage aux paiements, il est sans doute plus fécond de se demander pourquoi, là où existent les stocks alimentaires, on rencontre toujours d'autres biens $W$ qui ont occupé, immédiatement ou après un certain délai, une place prépondérante dans les paiements. La réponse se trouve probablement du côté de la sédentarité. Le lien entre sédentarité et stocks alimentaires est bien connu : sauf dans quelques rares cas de « conditions écologiques favorables ", le stockage alimentaire et la sédentarité représentent tout à la fois une condition et une conséquence l'un de l'autre. Or, la sédentarité est également une condition, sinon de l'existence des biens $W$, du moins de leur accumulation sur une échelle suffisante pour qu'ils puissent faire basculer les rapports sociaux vers les paiements et la richesse. Tant que l'élevage, cette forme de stock qui assure sa propre mobilité, n'est pas inventé, la sédentarité apparaît comme une condition nécessaire, quoique non suffisante, de la présence des biens $W$. Les sociétés structurées par les paiements forment un sous-ensemble au sein des sociétés sédentaires non éleveuses : il n'existait pas de nomades non éleveurs chez qui les paiements avaient été instaurés.

\section{Les biens W, condition nécessaire et suffisante des paiements?}

Les exemples des Asmat, des Calusa et des Jivaros indiquent que les paiements peuvent exister sans stockage alimentaire, tout en suggérant qu'en l'absence d'un tel stockage, ces paiements existent en raison de la présence de biens $W$. On peut cependant se demander jusqu'à quel point il est légitime de généraliser ces observations et de considérer à titre général que les biens $W$, sous réserve de se présenter en quantité suffisante, représentent une condition nécessaire de la transition vers les paiements.

Du point de vue de la logique formelle, il est difficile de prouver cette idée de 
manière définitive; on connaît le célèbre argument selon lequel des exemples qui vérifient une règle, si nombreux soient-il, n'en constituent jamais une véritable preuve. En pratique, toutefois, si la cohérence logique est un élément essentiel pour valider une loi sociale, son adéquation avec les observations - a fortiori lorsque celles-ci sont nombreuses - l'est tout autant. Si toutes les sociétés à richesse et sans stockage manipulent des biens $W$ et si, de surcroît, ces biens y jouent un rôle central, tant dans les processus de production que dans les paiements, notre hypothèse bénéficie à tout le moins d'une forte présomption favorable. Il suffirait certes, en sens inverse, de produire un seul exemple de société pratiquant les paiements sans qu'y existent des biens $W$ pour l'affaiblir, voire pour l'invalider. On ne peut écarter la possibilité qu'un tel exemple existe; cela paraît cependant très peu probable. Le nombre de sociétés connues qui conjuguent présence de la richesse et absence de stockage alimentaire est très réduit; il est donc permis de penser que si, parmi elles, certaines étaient dépourvues de biens $W$, elles auraient été assez aisées à identifier.

Reste la question symétrique : si la production ou l'acquisition de biens $W$ est une condition nécessaire du passage aux paiements, en constitue-t-elle également une condition suffisante? Autrement dit, l'existence de biens $W$ et l'existence de paiements sont-elles des propositions équivalentes? Une réponse positive à cette question signifierait que les sociétés sans paiements sont également nécessairement dépourvues (ou pourvues en trop faible quantité) de biens $W$. Il faut donc déterminer si cette proposition se vérifie pour les groupes concernés, qu'ils soient chasseurs-cueilleurs nomades ou cultivateurs.

Concernant ces derniers, une première objection serait que ceux-ci construisent toujours des habitations qui, si frustes soient-elles, représentent souvent un important investissement en travail; de même, à de rares exceptions près, le défrichage des jardins, fussent-ils temporaires, représente en l'absence d'outils de métal un effort harassant. C'est pourquoi, en définissant les biens $W$, on a pris la précaution de les réduire aux biens meubles. Pour remplacer une prestation en travail, des biens doivent être transférables. Ce n'est pas une simple question de droits de propriété : encore faut-il que l'usage effectif du bien puisse être transmis du gendre au beau-père et, audelà, à d'autres encore, le beau-père utilisant fréquemment la richesse reçue lors du mariage de ses filles pour permettre à ses propres fils de trouver des épouses. Une maison, un jardin pourraient à la rigueur être transférés au sein d'un même groupe local; mais on voit aisément que cette solution devient impraticable dès lors que les époux proviennent de groupes locaux différents.

Une autre série de contre-exemples pourrait être invoquée : celle de ces sociétés sans paiements qui investissent parfois un temps considérable dans la production de certains objets.

Pour l'Australie, on peut penser aux outils de pierre : c'est en effet sur ce continent, traditionnellement considéré comme techniquement retardataire, qu'ont été retrouvées les plus anciennes haches polies du monde. Ces haches ne remplissent cependant qu'assez mal les conditions requises pour déclencher un 
passage aux paiements. Comme, chez ces chasseurs-cueilleurs, elles n'étaient pas destinées à effectuer de gros travaux d'abattage, seul leur tranchant était poli, une opération qui prenait tout au plus quelques heures. Différentes expérimentations ont montré que "fabriquer les types australiens de haches polies, étant donné les matériaux, n'exigeait ni qualification particulière, ni un gros effort, ni beaucoup de temps ${ }^{48} »$.

Il est une autre production aborigène qui, en revanche, pouvait sans nul doute représenter un lourd investissement : celle des filets, de chasse ou de pêche (fig. 5), dont certains pouvaient atteindre plusieurs centaines de mètres de longueur $^{49}$. Selon divers observateurs ${ }^{50}$, leur fabrication demandait un travail considérable, ce que confirment les données disponibles. Ainsi, un filet à émeu conservé au South Australian Museum, qui mesure $52 \mathrm{~m} \times 0,8 \mathrm{~m}$, contient environ $350 \mathrm{~m}$ d'une corde de $5 \mathrm{~mm}$ de diamètre. Sur la base des informations données par W. Roth, son seul tressage nécessitait sans doute trois à quatre semaines de travail collectif. Les filets à oiseaux étaient encore plus exigeants, en raison de la finesse de leurs mailles. Un filet de 18 mètres sur 12, tel que le décrit Eyre ${ }^{51}$, aux mailles de $5 \mathrm{~cm}$, représentait environ 8000 mètres de corde! Celle-ci provenait de tendons ou de poils animaux, mais surtout de diverses fibres végétales (joncs, kurrajong, hibiscus...) et exigeait un long processus de traitement : il fallait souvent, entre autres, les tremper, les carder à la main ou aux dents, les sécher, les fouler, les peler et les mâcher. L'extraction de la fibre nécessitait parfois une cuisson ou une macération. Le

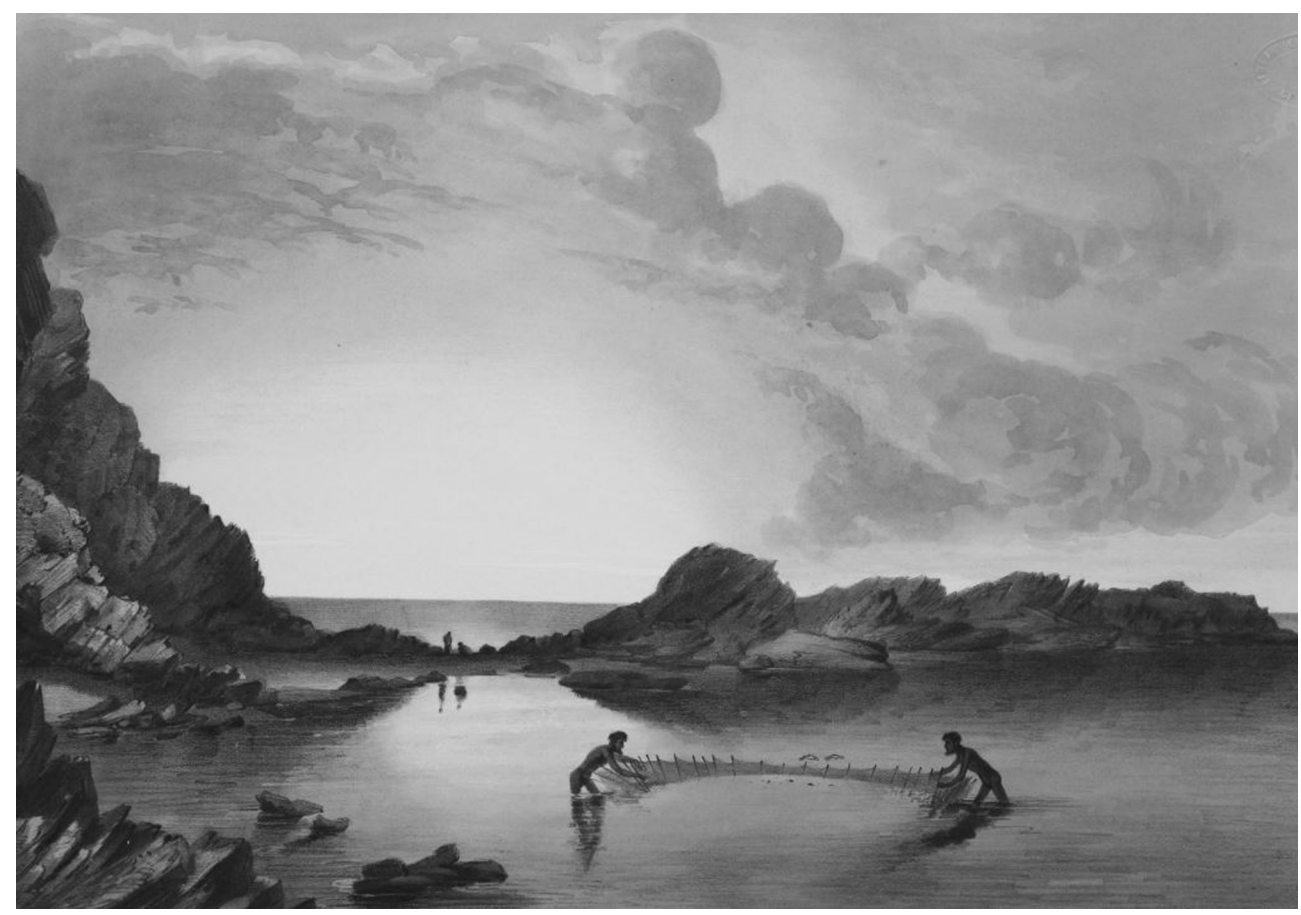

Figure 5. - Aborigènes pêchant au filet à Rapid Bay, Australie du sud (illustration des années 1840). 
tissage s'effectuait à l'aide de navettes et d'aiguilles de bois ${ }^{52}$.

Pourquoi les filets, bien qu'ils paraissent rassembler toutes les caractéristiques requises, $n^{\prime}$ ont-ils pas provoqué le passage aux paiements? On suggèrera que la raison tient à la fois à l'organisation du travail investi dans leur fabrication et au régime de leur propriété. Le travail nécessaire aux plus grands filets, destinés à la chasse, était le plus souvent collectif, impliquant le groupe entier. Dès lors, le filet, tout en constituant le cas échéant un article d'échange avec $l^{\prime}$ extérieur ${ }^{53}$, ne pouvait guère devenir le moyen de solder des obligations internes au groupe; voilà pourquoi on a précisé précédemment que, pour être un bien $W$, un objet devait résulter des efforts d'un individu ou un petit groupe d'individus. Les filets de dimensions plus modestes, en particulier ceux destinés à la pêche, étaient certes fabriqués individuellement. En lui-même, le fait que le plus gros du travail, à savoir la collecte et la préparation des fibres, incombait aux femmes ${ }^{54}$, ne saurait être invoqué : dans les Hautes Terres de Nouvelle-Guinée, le porc, qui représente la richesse par excellence, résultait d'un travail essentiellement féminin. Mais - et c'est sans doute la différence pertinente avec les filets australiens - ce sont les hommes qui avaient des droits de propriété sur les porcs, alors que chez les Aborigènes, les filets confectionnés par les femmes leur appartenaient. Le prix de la fiancée étant par définition un versement effectué par les hommes et à destination d'autres hommes, il y avait là, semble-t-il, un obstacle supplémentaire à la transformation des filets en moyens de paiement.
Concluons en précisant, si nécessaire, $q^{\prime}$ 'on ne propose pas de verser dans un déterminisme matérialiste caricatural et d'établir une équation trop stricte entre les caractéristiques techno-économiques d'une société et le passage aux paiements. Sans doute certains biens $W$ peuvent-ils être identifiés dans les sociétés sans richesse. Notre hypothèse est qu'il existe un effet de seuil, à partir duquel leur importance rend la transition de plus en plus probable. Cette possibilité tend à faire défaut chez des nomades dépourvus de moyens de transport et qui économisent en permanence sur leur équipement. Inversement, la sédentarité a sans doute joué un rôle décisif, non en faisant naître les biens $W$, mais en permettant leur accumulation dans une quantité qui a entraîné certaines transformations sociales qualitatives. Si cette conjecture se vérifiait, la transition aux paiements n'aurait donc pas été le fruit de l'apparition d'un phénomène technico-économique nouveau, mais de sa croissance au-delà d'un certain seuil (susceptible, bien sûr, d'une certaine variation d'une société à l'autre). 


\section{Conclusion}

L'hypothèse selon laquelle ce sont les biens $W$ qui engendrent le basculement vers les paiements et la richesse semble être celle qui rend compte de la manière la plus satisfaisante des données ethnographiques. Elle intègre les cas rares, mais nettement identifiés, de sociétés qui, tout en ignorant le stockage alimentaire, connaissent néanmoins les paiements et, par conséquent, un degré plus ou moins prononcé de différenciation sociale, et elle ne se heurte a priori à aucune exception connue. Formulée dans un vocabulaire marxiste, la transition aux paiements se lit alors comme la première victoire, dans l'histoire des rapports sociaux, du travail mort sur le travail vivant.

Les contours de cette loi sociale exigent certes d'être affinés : le caractère suffisant de la présence des biens $W$ pour le passage aux paiements, en particulier, devra faire l'objet de recherches plus approfondies. Un autre angle mort concerne le wergeld, qui ne peut apparaître immédiatement comme l'équivalent d'un travail mais comme celui d'une vie. On sait toutefois que, même si, dans les détails, prix de la fiancée et wergeld ne coïncident pas nécessairement en tout point, ces deux phénomènes sociaux apparaissent très fortement corrélés. Notre intuition est que l'explication du prix de la fiancée par les biens $W$ (donc, par des phénomènes relevant de la sphère du travail) constitue également une voie féconde pour élucider les voies de la mise en place du wergeld. C'est aussi, semble-t-il, ce qui ressort de la belle étude comparative menée sur les sociétés néo-guinéennes par Pierre Lemonnier qui avançait notamment que « la comparaison du statut symbolique du porc dans ces deux régions de Nouvelle-Guinée [les Highlands et la côte méridionale] conduit à formuler $l^{\prime}$ hypothèse que la valeur de substitut de vie que l'on attribue - ou pas - à l'animal est corrélée avec la part de travail [...] impliquée dans sa croissance et dans sa reproduction ${ }^{55} »$.

En l'état, et même dans sa version la plus prudente, l'hypothèse des biens $W$ permet d'améliorer la cohérence entre nos conceptions et nos observations; elle ouvre également la possibilité que certaines sociétés disparues, dont l'archéologie indique un certain niveau de sédentarité sans toutefois déceler d'indices probants de stockage alimentaire, puissent avoir d'ores et déjà connu la transition vers les paiements et les inégalités - on pense, par exemple, aux cas $\mathrm{du}$ Natoufien et du Mésolithique côtier d'Europe de l'Ouest; mais ceux du Magdalénien, du Pavlovien d'Europe centrale, du Gravettien et du Mézinien russes, avec leurs productions matérielles élaborées, mériteraient eux aussi d'être examinés sous cette lumière. Plus encore, si la version forte de notre hypothèse se voyait étayée, c'est-à-dire si l'examen de l'ensemble des sociétés sans richesse connues confirmait l'équivalence entre un certain niveau de biens $W$ et la présence de paiements, l'archéologie disposerait d'un critère discriminant qui lui permettrait, avec une marge d'erreur réduite, de considérer la présence de la richesse dans de telles sociétés non comme une simple éventualité, mais comme une relative certitude ${ }^{56}$. 


\section{Notes}

1. Vere Gordon CHilde, Man makes himself, Londres, Watts \& Co, 1948 [1936].

2. Harry W. Pearson, "The economy has no surplus : critique of a theory of development ", in Karl Polanyi, Conrad M. Arensberg, Harry Pearson (éd.), Trade and market in the early empires; economies in theory and history, Glencoe, The Free Press, 1957; Marvin HARRIs, " The economy has no surplus? ", American Anthropologist, New Series, 61-2, 1959, p. 185-199; George Dalton, "A note of clarification on economic surplus ", American Anthropologist, New Series, 62-3, 1960, p. 483-490; id., " Economic surplus, once again », American Anthropologist, New Series, 65-2, 1963, p. 389-394; Abraham Rotstein, « A note on the surplus discussion ", American Anthropologist, New Series, 63-3, 1961, p. 561-563; Martin Orans, "The surplus ", Human Organization, 2, 5, 1966, p. 24-32.

3. Alain Testart, Les chasseurs-cueilleurs ou l'origine des inégalités, Paris, Société d'ethnographie (Université Paris 10-Nanterre), 1982.

4. Alain Testart, Éléments de classification des sociétés, Paris, Errance, 2005.

5. Nous poursuivons là un questionnement entrepris depuis plusieurs années. Une version préliminaire a fourni la matière de l'exposé du 2 avril 2016 au séminaire Marx au XXI siècle, dirigé par Jean Salem (Université Paris 1).

6. Alain Testart, Avant l'histoire : l'évolution des sociétés, de Lascaux à Carnac, Paris, Gallimard, 2012, p. 357.

7. Dale Hutchinson et al., "The Calusa and prehistoric subsistence in central and south Gulf Coast Florida », Journal of Anthropological Archaeology, 41, 2016, p. 55-73.

8. Hernando Fontaneda, Memoir of Hernando de Escalante Fontaneda, Washington, 1854 [1575], p. 16.

9. John Goggin, William Sturtevant, "The Calusa : a stratified non-agricultural society (with Notes on sibling marriage) ", in Ward H. Goodenough (éd.), Explorations in cultural anthropology : essays in honor of George Peter Murdoch, New York, McGraw-Hill, 1964, p. 188194.

10. Alain Testart, La servitude volontaire. 1 : Les morts d'accompagnement; 2 : L'origine de l'État, Paris, Errance, 2004.

11. Gerard A. ZegwaART, « Headhunting practices of the Asmat of Netherlands New Guinea ", American Anthropologist, New Series, 61-6, 1959, p. 1020-1041.
12. David B. Eyde, Cultural correlates of warfare among the Asmat of South-West New Guinea, Ph. D. of philosophy, Yale University, 1967, p. 64.

13. Ibid.

14. Paul B. RosCoE, «The hunters and gatherers of New Guinea », Current Anthropology, vol. 43, 1, 2002, p. 157.

15. D. B. Eyde, Cultural correlates of warfare..., op. cit., p. 38; Patricia K. TownSEnd, " Sago production in a New Guinea economy ", Human Ecology, vol. 2, 3, 1974, p. 225.

16. Ibid., p. 337.

17. FranckTrenkenshum, An Asmat Sketch Book, vol. 1, Hastings, Asmat Museum of Culture and Progress; Djajapura, Franciscan Press, 1970, p. 58.

18. Alphonse Sowada, Socio-economic survey of the Asmat peoples of Southwestern New Guinea, Washington, Catholic University of America, 1961, p. 57.

19. D. B. EYDE, Cultural correlates of warfare..., op. cit., p. 337; F. TrenKenshum, An Asmat Sketch Book..., op. cit., p. 51; A. SowAdA, Socio-economic survey of the Asmat peoples, op. cit., p. 84-87.

20. Paul Van Arsdale, An Asmat Sketch Book $n^{\circ} 5$ : perspective on development in Asmat, Agats, The Asmat Museum of Culture and Progress, 1975, p. 36.

21. Gerard A. ZegwaARd et J. H. M. C. BoelaARs, « De Sociale Structuur van de Asmatbevolking ", Adatrechtbundel XLV, p. 298, cité par A. Sowada, Socio-economic survey of the Asmat peoples, op. cit., p. 84.

22. A. SowADA, op. cit., p. 53-55.

23. D. B. Eyde, Cultural correlates of warfare..., op. cit., p. 333.

24. Gerard A. ZegwaARD, « Headhunting practices of the Asmat of Netherlands New Guinea », American Anthropologist, New Series, vol. 61, 6, 1959, p. 1040.

25. D. B. EYDE, Cultural correlates of warfare..., op. cit., p. 233.

26. Bruce M. Knauft, South Coast New Guinea cultures; history, comparison, dialectic, Cambridge, Cambridge University Press, 1993, p. 74.

27. F. TrenKenshuH, An Asmat Sketch Book..., op. cit., p. 31; D. B. EYDE, Cultural correlates of warfare..., op. cit., p. 221.

28. Vincent Van Amelsvoort, Culture, stone-age and modern medicine : the early introduction of integrated rural health in a non-literate society, Assen, Van Gorcum, 1964, p. 42.

29. D. B. EYdE, Cultural correlates of warfare ..., op. cit., p. 228; également F. TrenKenshum, An Asmat Sketch Book..., op. cit., p. 31; V. VAN AMELsvoorT, Culture, stone-age and modern medicine..., op. cit., 
p. 43 ; P. VAN Arsdale, An Asmat Sketch Book $n^{\circ} 5 \ldots$, op. cit., p. 50 .

30. Alain Testart, Éléments de classification des sociétés, Paris, Errance, 2005.

31. Pierre Lemonnier, Guerres et festins. Paix, échanges et compétition dans les Highlands de Nouvelle-Guinée, Paris, Éditions de la Maison des Sciences de l'Homme, 1990.

32. Christophe Darmangeat, Le communisme primitif n'est plus ce qu'il était. Aux origines de l'oppression des femmes, $2^{\mathrm{e}}$ éd. Toulouse, Smolny, 2012, p. 236.

33. A. Testart, Éléments de classification des sociétés, op. cit., p. 37-38.

34. Ibid.

35. Pierre Petrequin, Anne-Marie Petrequin, Écologie d'un outil : la hache de pierre en Irian Jaya (Indonésie), Monographie du C.R.A. (12), 2e éd. Complétée, Paris, Éditions du CNRS, 2000, p. 377.

36. A. SowAdA, Socio-economic survey of the Asmat peoples, op. cit., p. 46.

37. D. B. EydE, Cultural correlates of warfare..., op. cit., p. 45.

38. Ibid., p. 53.

39. Richard SAlisbury, From stone to steel. Economic consequences of a technological change in New Guinea, Melbourne, Melbourne University Press, 1962; William H. TownsEND, «Stone and steel tool use in a New Guinea society », Ethnology, VIII (2), 1969, p. 199-205; Maurice Godelier, José GARANGER, "Outils de pierre, outils d'acier chez les Baruya de Nouvelle-Guinée ", L'Homme, 13 (3), 1973, p. 187-220.

40. P. Van Arsdale, An Asmat Sketch Book $n^{\circ} 5 \ldots$, op. cit., p. 36 .

41. D. B. EydE, Cultural correlates of warfare..., op. cit., p. 43.

42. Carlos Fausto, David Rodgers. « Of enemies and pets : warfare and shamanism in Amazonia ", American Ethnologist, vol. 26 (4), 1999, p. 933-956.

43. Michael Harner, Les Jivaros, Paris, Payot, 2006, p. 91.

44. Ibid., p. 190.

45. Alain Testart, « L'évolution des chasseurscueilleurs : hypothèse supplétive sur le mariage »,
Bulletin de la Société préhistorique française, vol. 111, 4, 2014, p. 593-602.

46. Ibid., p. 597.

47. Ibid., p. 598.

48. Franck P. Dickson, "Ground Edge Axes », Mankind, 8, 1973, p. 211.

49. Leonn SAtTerthwAit, "Socioeconomic implications of Australian Aboriginal net hunting ", Man, New Series, vol. 22, 4, 1987, p. 613-636.

50. Gerard KREFFT, « On the manners and customs of the Aborigines of the Lower Murray and Darling ", Transaction of the Philosophical Society of New South Wales, 1865, p. 361-362; Walter E. Rотн, Food : its search, capture, and preparation, North Queensland Ethnography, Bulletin $\mathrm{n}^{\circ}$ 3, 1901, p. 28; Alice M. DunCAN-KemP, Our sandhill country : nature and man in south-western Queensland, Sydney, Angus \& Robertson, 1933, p. 122.

51. John EYRE, Journals of expeditions of discovery into Central Australia, and overland from Adelaide to King George's Sound, in the years 1840-1, Londres, T. \& W. Boone, 1845, t. 2, p. 286.

52. Leonn SATterthwAit, « Aboriginal Australian net hunting ", Mankind, vol. 16, 1, 1986, p. 31-48.

53. G. KREFFT, «On the manners and customs of the Aborigines of the Lower Murray and Darling », op. cit., p. 361.

54. Gerard KREFFT, «Food : its search, capture, and preparation ", North Queensland Ethnography, Bulletin $n^{\circ} 3$, 1901, p. 22; Leonn SATterthwait, communication personnelle.

55. Pierre Lemonnier, « Le porc comme substitut de vie; formes de compensation et échanges en Nouvelle-Guinée ", Social Anthropology 1, 1A, 1993, p. 49.

56. Je tiens à remercier Guillaume Carnino, Maurice Fhima, Pierre Lemonnier, Jean-Marc Pétillon et Boris Valentin pour l'ensemble de leurs remarques stimulantes sur ce texte. Selon la formule consacrée, les erreurs qu'il pourrait comporter relèvent de ma seule responsabilité. 